

\section{REVISTA \\ TEORÍA Y PRÁCTICA \\ DE LA \\ ARQUEOLOGÍA HISTÓRICA LATINOAMERICANA}

ISSN: 2250-866X (impreso) | ISSN: 2591-2801 (en línea)

AÑo VIII, VOLUMEN 8, PRIMAVERA DE 2019

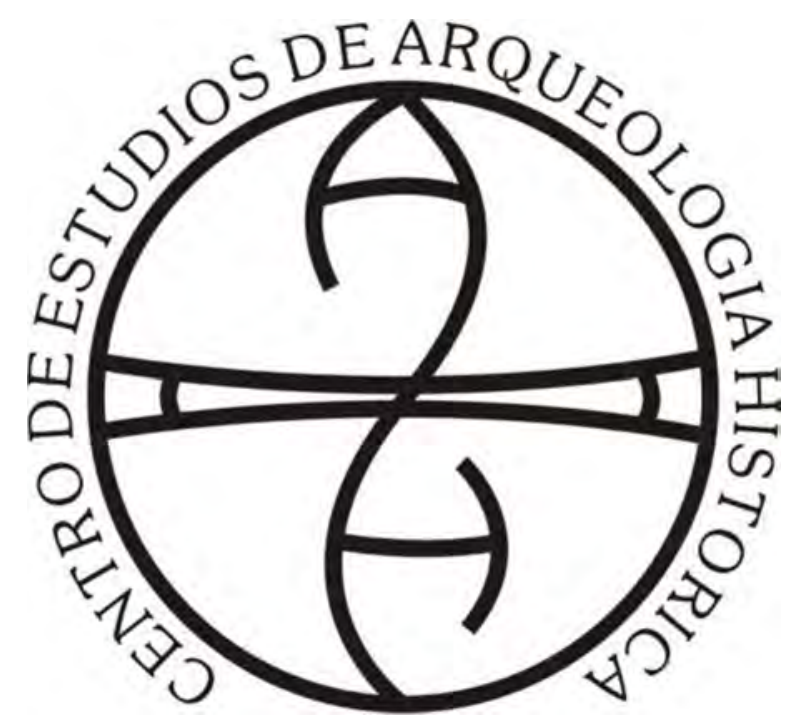

Centro de Estudios de Arqueología Histórica

FACULTAD DE HUMANIDADES Y ARTES | UNIVERSIDAD NACIONAL DE ROSARIO 
(Universidad Nacional de Rosario, Universidad Nacional de Río Cuarto,

Universidad Nacional de San Juan, Universidad de la República, Universidad Nacional de Trujillo)

\author{
AUTORIDADES DE LA UNIVERSIDAD NACIONAL DE ROSARIO \\ RECTOR: Lic. Franco Bartolacci \\ VICE-RECTOR: Od. Darío Macía \\ SECRETARIO GENERAL: Prof. José Goity \\ SECRETARIA ACADÉMICO Y DE APRENDIZAJE: Dr. Marcelo Vedrovnik \\ SECRETARÍA DE CIENCIA TECNOLOGÍA E INNOVACIÓN \\ PARA EL DESARROLLO: Ing. Guillermo Montero.
}

\author{
AUTORIDADES DE LA FACULTAD DE HUMANIDADES Y ARTES \\ DECANO: Prof. Alejandro Vila \\ VICEDECANA: Prof. Marta Varela \\ SECRETARIA ACADÉMICA: Dra. Marcela Coria
}

\author{
AUTORIDADES DEL CENTRO DE ARQUEOLOGÍA HISTÓRICA \\ DIRECTORA: Dra. Ana Rocchietti \\ SECRETARIA: Prof. Nélida de Grandis \\ PROSECRETARIA: Lic. Marianela Bizcaldi
}

DIRECTORAS - EDITORAS:

Dra. Ana Rocchietti y Prof. Nélida De Grandis

SECRETARIA DE EDICIÓN: Dra. Irene Dosztal

Este número es co-edición de las ponencias

del VIII Congreso Nacional de Arqueología Histórica (2018) entre:

Centro de Estudios en Arqueología Histórica: Directora Ana Rocchietti

Centro de Estudios en Arqueología Regional: Director Fernando Oliva

Centro de Estudios en Arqueología Subacuática: Directora Mónica Valentini

Departamento de Arqueología, Escuela de Antropología: Director Fernando Oliva
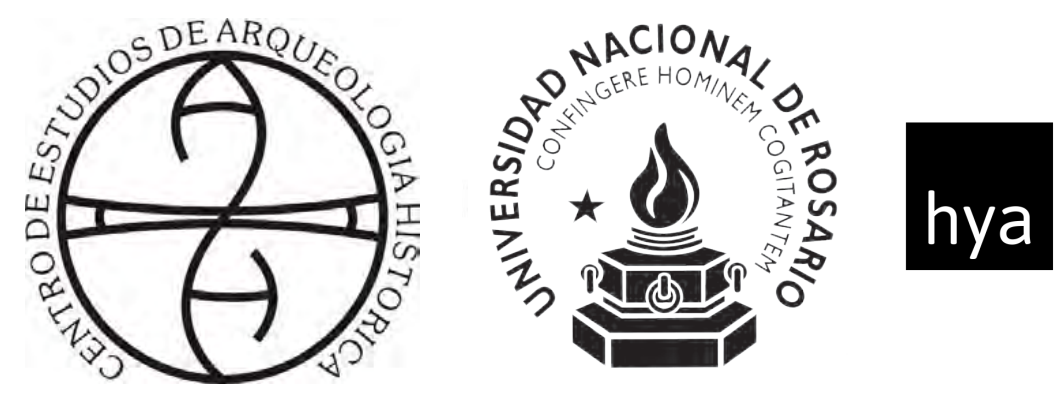

Facultad de Humanidades

y Artes_UNR
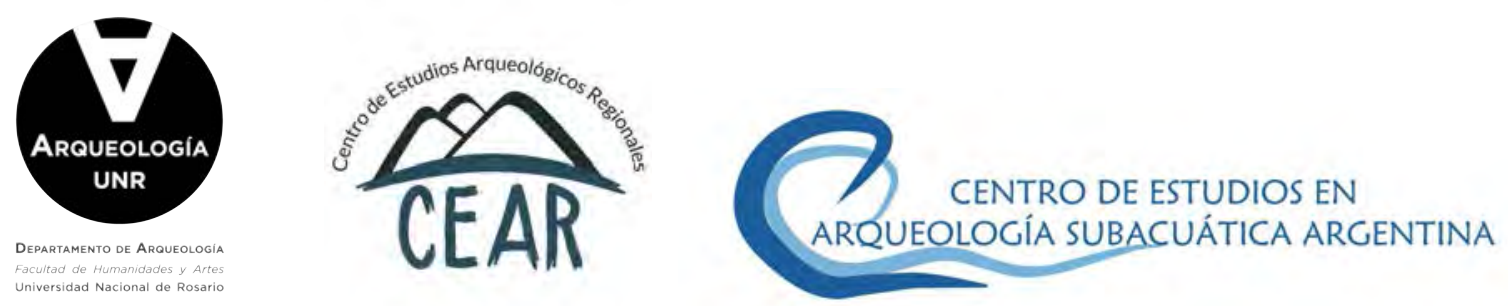
Comité Permanente de los Congresos Nacionales de Arqueología Histórica

Dr. Daniel Schávelzon (Universidad Nacional de

Buenos Aires)

Prof. María Teresa Carrara (Universidad Nacional de Rosario)

Prof. Carlos Baldassarre (Museo Municipal de Río

Grande, Tierra del Fuego) in memoriam

Dr. Mariano Ramos (Universidad Nacional de Luján, CONICET)

Dr. Horacio Chiavazza (Universidad Nacional de Cuyo)

Dra. Ana María Rocchietti (Universidad Nacional de

Rosario, Universidad Nacional de Río Cuarto)

Lic. Facundo Gómez Romero (Universidad Autónoma

de Barcelona)

\section{Comité Científico}

Dra. Tânia Andrade Lima (Universidade Federal do Rio de Janeiro)

Prof. Réginald Auger (CELAT/Département des

Sciences Historiques, Université Laval, Canadá)

Dr. Roberto Bárcena (Universidad Nacional de Cuyo, CONICET)

Dra. Marta Bonaudo (Universidad Nacional de Rosario, CONICET)

Dr. Leonel Cabrera (Universidad de la República, Uruguay)

Dr. Luis María Calvo (Universidad Católica de Santa

$\mathrm{Fe})$

Prof. Juan Castañeda Murga (Universidad Nacional de

Trujillo, Perú)

Dr. Carlos Ceruti (Museo de Ciencias Naturales y

Antropología "Prof. Antonio Serrano". Paraná)

Dr. Horacio Chiavazza (Universidad Nacional de Cuyo)

Dra. Silvia Cornero (Universidad Nacional de Rosario)

Prof. Pedro Paulo Funari (Universidade Estadual de

Campinas, Brasil)

Lic. Jorge A. Gamboa Velásquez (Universidad Nacional

Santiago Antuñez de Mayolo, Perú)

Dr. Eduardo Alejandro García (Universidad Nacional de

San Juan, CONICET)

Prof. Nélida De Grandis (Universidad Nacional de

Rosario)

Dr. Juan Bautista Leoni (Universidad Nacional de

Rosario, CONICET)

Dra. Amancay Martínez (Universidad Nacional de San

Luis)

Dra. Catalina Teresa Michieli (Universidad Nacional de

San Juan, CONICET)

Lic. Fernando Oliva (Universidad Nacional de Rosario)

Ing. Adrián Pifferetti (Universidad Tecnológica
Nacional Regional Rosario)

Dr. Mariano Ramos (Universidad Nacional de Luján, CONICET)

Dra. Ana María Rocchietti (Universidad Nacional de Rosario)

Dr. Daniel Schávelzon, (Universidad Nacional de Buenos

Aires, CONICET)

Dra. Carlota Sempé (Universidad Nacional de La Plata)

Dr. Mario Silveira (Universidad Nacional de Buenos Aires)

Dra. Silvia Simonassi (Universidad Nacional de Rosario)

Dra. Alicia Tapia (Universidad Nacional de Buenos Aires, Universidad Nacional de Luján)

Lic. Mónica P. Valentini (Universidad Nacional de Rosario)

Agrim. Benito Vicioso (Universidad Nacional de Rosario)

\section{Evaluaron este volumen}

Roberto Bárcena, María Teresa Boschin, Leonel Cabrera, Ulises Camino, María Rosa Carbonari, Carlos Ceruti, Horacio Chiavazza, Nicolás Ciarlo, Silvia Cornero, Eduardo Crivelli, Javier García Cano, Martín Gentinetta, María Laura Gili, Carlos Landa, Matilde Lanza, Melina Malandrino, Sebastián Pastor, Victoria Pedrotta, Josefina Piana,

Mercedes Podestá, Mariano Ramos, Daniel Schavelzon, Diana Tamburini, Mónica Therrien, Mónica Valentini y María Teresa Boschin

\section{Diseño y diagramación}

Eugenia Reboiro

(eugenia.reboiro@gmail.com)

\section{Curadoría}

Ana Rocchietti e Irene Dosztal

Foto de tapa: imagen del texto de Croce.

\section{Propietario responsable:}

Facultad de Humanidades y Artes, Universidad Nacional de Rosario Centro de Estudios de Arqueología Histórica

Entre Ríos 758. Rosario, provincia de Santa

Fe (2000). Argentina.

Telf.: +54 (0341) 4802670

E-mail: ceahunr@gmail.com

Decreto Ley 6422/57 de Publicaciones

Periódicas 


\section{Índice}

Editorial.

Arqueología histórica: programa de investigación y dimensiones epistemológicas...9 Ana María Rocchietti

De lozas, iglesias y machaqueños. Primeros pasos hacia una arqueología histórica en la cuenca norte del río desaguadero (La Paz, Bolivia).

Salvador Arano Romero

Identificación arqueológica de acciones militares en el campo de batalla de Cepeda, 1859.

Juan B. Leoni, Lucas H. Martínez, Cecilia Arias Morales, Daniela Cadenas,

Faustino Godoy, Mauro Ganem, María de la Paz Blanche y Héctor Meletta

Para una historia de la arqueología urbana en Buenos Aires (1848-1910) .59

Daniel Schávelzon y Francisco Girelli

Definiendo la cultura material colonial a partir de colecciones arqueológicas del Museo de La Plata.

Melina Bednarz, Julieta Penesis y Ana Igareta

La tangibilidad del conflicto: Arqueología del presente en las fronteras del norte santafesino....

Paula Del Río y Silvia Cornero

Arqueología histórica: evidencia material en el paisaje del establecimiento jesuita "La saladilla” (quebrada de Saladillo, La Rioja, Argentina). Juan Carlos Giuliano, Maira Ayelén Carrizo y María Soledad Gheggi

Graffiti ¿evidencia arqueológica o acto vandálico?. Camila Oliva

Arqueología histórica de los sistemas hidráulicos de la Hacienda

Jalpa de Cánovas, Guanajuato, México, y su relación con el sistema-mundo, Siglos XVIII-XIX

José Alberto Aguirre Anaya y Edgar Quispe Pastrana 
Paseo del bajo: una trinchera en la historia de Buenos Aires.................................143 Federico Martín Croce

Diferentes concepciones del patrimonio y su aplicación a la Casa Histórica de Tucumán también llamada Casa de la Independencia........................................155 Guillermo Etchevers

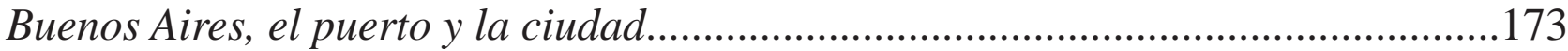
Mónica P. Valentini y Javier García Cano 


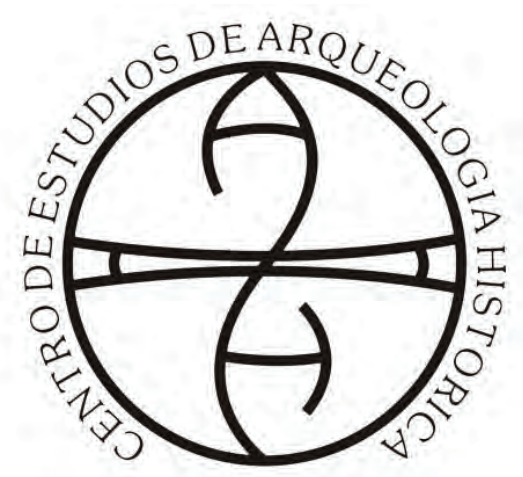

Centro de Estudios de Arqueología Histórica

Universidad Nacional de Rosario
Teoría y Práctica de la Arqueología Histórica

Latinoamericana | Año VIII. Vol. 8 | 2019

Revista del Centro de Estudios de Arqueología

Histórica, Facultad de Humanidades y Artes,

Universidad Nacional de Rosario

https://teoriaypracticaah.unr.edu.ar/index.php/index

ceahunr@gmail.com

ISSN en línea: 2591-2801

ISSN versión impresa: 2250-866X

\title{
DEFINIENDO la CULTURA MATERIAL COLONIAL A PARTIR DE colecciones arqueológicas del Museo de la Plata
}

\author{
Melina Bednarz *, Julieta Penesis ** y Ana Igareta ***
}

\section{Resumen}

Desde hace más de un siglo, las colecciones arqueológicas del Museo de La Plata sirven como material de referencia y comparación para investigadores locales y del exterior. Y aunque suele asumirse que las piezas dan cuenta del desarrollo cultural prehistórico del país, tareas de puesta en valor realizadas en la última década pusieron en evidencia que dichas colecciones incluyen también un importante corpus de piezas históricas, en su mayoría coloniales, que nunca habían sido reconocidas como tales. En parte, tal desconocimiento deriva de la escasez de información contextual con que cuentan los materiales pero se relaciona también con la falta de estudios sistemáticos de las características físicas de las piezas reunidas en muchas de las colecciones.

En el marco de un trabajo más amplio de elaboración de un primer catálogo de referencia de dicho conjunto colonial, se propuso-a partir de la revisión de una muestra del material en cuestión- la construcción de criterios discretos que sirvieran como herramienta de base para la identificación de piezas históricas que pudieran verse afectadas por una situación semejante de ausencia de información contextual.

Palabras clave: colecciones arqueológicas- cultura material - periodo colonial

\begin{abstract}
For more than a century, the archaeological collections of the La Plata Natural Sciences Museum have served as reference for both local and foreign researchers, and although it is usually assumed that every item reflects part of the prehistory of our country, through our work in collections management we came to realize that there were many historical artifacts - most of them colonial- that had never been identified as such.

While the general aim of our project is to build a catalogue of colonial artifacts, this paper aims at explaining the process that we developed to define the criteria that allowed us to identify historical artifacts lacking contextual information.
\end{abstract}

Keywords: archaeological collections - material culture - colonial period

* CONICET - CAU, FCNyM, UNLP, meliarqueo@gmail.com

** FCNyM, UNLP, jpenesis@gmail.com

*** CONICET - HiTePAC, FAU- FCNyM - UNLP, aigareta@gmail.com 
Teoría y Práctica de la Arqueología Histórica Latinoamericana | Año VIII. Vol. 8 | 2019

ISSN en línea: 2591-2801 | ISSN versión impresa: 2250-866X

\section{Consideraciones generales}

A lo largo del tiempo, las colecciones arqueológicas del Museo de La Plata han sido fuente de referencia ineludible para numerosas investigaciones desarrolladas en el país y en el exterior, tanto por la abundancia y diversidad de los conjuntos reunidos en ellas como por la extensión del lapso que abarcan (Collazo, 2012). Las tareas de puesta en valor llevadas a cabo desde el año 2008 en el Depósito 25 de la División Arqueología del Museo (DA de aquí en adelante) pusieron en evidencia que dichas colecciones incluyen un importante corpus de piezas históricas-en su mayoría coloniales- que nunca habían sido identificadas como tales (Igareta y Vargas Gariglio, 2016). El reconocimiento de ese primer conjunto de piezas dio comienzo a un trabajo sistemático de clasificación a través del cual buscamos reconocer y registrar sus características en detalle, sistematizándolas luego en un inventario a modo de catálogo que pueda servir como herramienta de identificación de conjuntos semejantes.

Apenas iniciado el trabajo nos encontramos con el problema de que, una vez separadas aquellas piezas con rasgos diagnósticos claramente reconocibles como históricos -materia prima, morfología y tecnología de manufactura foráneas-habíamos reconocido como históricas a otro conjunto cuyas características resultaban más difíciles de aprehender y clasificar. Consideramos entonces la necesidad de definir criterios sintéticos que sirvieran como herramientas para la identificación sistemática de tales objetos (Igareta, Bednarz y Penesis, 2018) y nos permitieran ir más allá del reconocimiento de su cronología basado en nuestra propia familiaridad con objetos semejantes. Cabe recordar que tanto la falta de información contextual ${ }^{1} \mathrm{y}$ de referencia que afectan a gran parte de las colecciones arqueológicas del Museo, como las dificultades de realizar análisis invasivos y/o destructivos sobre dichos conjuntos limitan las posibilidades de estudio directo del material, por ejemplo para la obtención de fechados absolutos. Por ello se optó, en cambio, por desarrollar un análisis comparativo de las piezas y sus características a partir de referentes conocidos actuales y/o arqueológicos como herramienta de asignación de antigüedad.

\section{Algunos datos sobre las colecciones del Depósito 25}

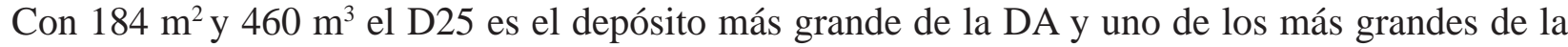
institución, almacenando un estimado de 130.000 piezas enteras entre las que se incluyen las de las llamadas colecciones fundacionales, materiales donados por F. Moreno para la inauguración del Museo en 1884 y colectados y/o donados por otros investigadores entre fines del siglo XIX y 1930.

Durante los trabajos de acondicionamiento y puesta en valor de algunas de ellas -la Colección Francisco Moreno, la Samuel Lafone Quevedo, la Adolfo Methfessel, la Joaquín V. González, la Carlos Bruch, la Fernando Márquez Miranda- reconocimos un primero conjunto de elementos de origen histórico asociados a restos arqueológicos más antiguos. Piezas semejantes fueron identificadas ocasionalmente en la Colección Benjamín Muníz Barreto, que se encuentra en guarda en otro depósito de la División, pero aún se encuentra pendiente una revisión sistemática de dicho conjunto. En muchos casos, las fichas de inventario del material que reconocimos como histórico -realizadas durante las primeras décadas del siglo XX- dan cuenta de la extrañeza que sus características generó en su colector o en quien organizó luego la colección en el Museo; indicaciones tales como "objeto de uso desconocido" para un cuchillo o una punta de lanza perfectamente conservados, o el registro de brillantes cuentas de vidrio azul como “cuentas de piedra” muestran hasta qué punto estas piezas generaron confusión.

Como ya se ha mencionado, si bien muchas de las colecciones del D25 poseen escasa información de referencia sobre la procedencia del material y casi nulo soporte documental, gráfico y/o fotográfico 
que dé cuenta de las condiciones de hallazgo, otras fuentes de información tales como estudios científicos previos, los relatos de época de sus colectores ${ }^{2} y$ las características intrínsecas de ciertos elementos (urnas funerarias y restos esqueletarios) han permitido estimar que un porcentaje mayoritario de las piezas procede de cementerios(Bruch, 1911;Chiappe, 1962; entre muchos otros).

\section{Proceso de construcción de criterios para identificar piezas históricas}

Como señalamos, el proceso de identificación de la presencia de restos históricos comenzó de modo espontáneo con el hallazgo de piezas elaboradas con materiales no utilizadas en el continente americano antes de la llegada de los europeos. Sin embargo, luego se hizo evidente que las materias primas novedosas no eran criterio suficiente para abarcar todos los elementos que habíamos incluido en nuestra muestra de piezas históricas, por lo que se decidió considerar otros criterios que nos permitieran registrar, de modo sistemático y reproducible, los rasgos que fuimos definiendo como diagnósticos. De esta manera a la identificación de materias primas sumamos otras variables simples como las técnicas de manufactura, la morfología, la decoración y por último la funcionalidad. Resulta interesante mencionar que el primer proceso de reconocimiento de las piezas se basó en nuestra experiencia como investigadores en el análisis de materiales históricos recuperados en contextos de excavación, por lo que resultó un interesante desafío el proponernos sistematizar ese saber y aplicarlo al estudio de materiales de colección.

Para la construcción de la muestra se consideraron piezas enteras o con un muy alto grado de integridad para minimizar posibles interpretaciones erróneas, excepto en aquellos casos en que las materias primas nos permitían asegurar su carácter histórico (eg. cuentas de vidrio partidas o fragmentos de hojas de cuchillo de hierro).Las materias primas identificadas en todo el conjunto fueron piedra, vidrio, hierro, cerámica (tanto oxidante como reductora, con o sin presencia de mica), bronce y madera.

\section{Tabla 1}

\section{Cantidad de elementos incluidos en la muestra contabilizados por materia prima}

\begin{tabular}{lllllll}
\hline Material & Vidrio & Cerámica & Hierro & Bronce & Madera & Lítico \\
\hline Total & 1052 & 99 & 38 & 13 & 2 & 3 \\
\hline
\end{tabular}

Al considerarlas técnicas de manufactura de las piezas seleccionadas nos encontramos con distintos escenarios posibles en cuanto a su carácter diagnóstico. En el primero de ellos la identificación de técnicas novedosas para el continente (eg.:uso de torno o vidriados en superficie) nos permitió asignar cronológicamente con precisión relativa las piezas a momentos históricos. En el segundo, el reconocimiento de que las técnicas de manufactura locales tuvieron continuidad a lo largo de toda la época colonial, nos enfrentó a la posibilidad de hallarnos frente a elementos producidos durante el periodo histórico pero que, ante la ausencia de rasgos novedosos y por la mencionada carencia de información contextual, no nos es posible asignar a un período determinado. El tercer escenario posible es el representado por manufacturas locales cuyos rasgos considerados por separado no permiten una asignación cronológica precisa pero que presentan algún elemento o combinación novedosa de elementos -por ejemplo forma y decoración- que al ser considerados de manera articulada permite postular una adscripción cronológica general. 
En lo que refiere a la morfología de los materiales incluidos en la muestra, cabe mencionar que en algún momento estimamos que, considerados de modo aislado, algunos de sus componentes-asas, bases, bordes, etc.- podían servir en sí mismos como elementos diagnósticos de cronología, pero la consulta con especialistas y con bibliografía sobre el tema nos obligó a reconsiderar tal propuesta. Luego, estimamos que el grado de certeza en la asignación temporal de las piezas se incrementa cuando los rasgos son analizados de manera combinada y que es la articulación de los rasgos entre sí más que su presencia o ausencia lo que determina sus posibilidades informativas.

Por su parte, la ornamentación observada en las piezas que identificamos como históricas y las dificultades halladas al momento de intentar sistematizar sus características nos convenció de la necesidad de aceptar la propuesta elaborada como provisoria y sujeta a permanente revisión. El punto de partida para este criterio fue el reconocimiento de que ciertos objetos históricos presentan motivos -rasgos floreales, palabras o fechas- que no se corresponden con diseños registrados en las tradiciones americanas prehispánicas. Por otra parte, tomamos en cuenta la presencia de elementos que han sido atribuidos por los investigadores a contextos coloniales -Caspinchango, Quinchamalí- no estrictamente por la presencia de ciertos rasgos sino por el modo el que estos rasgos se articulan entre sí.

Por último, en lo que respecta al criterio de funcionalidad -y recordando las mencionadas limitaciones implícitas en no poder realizar análisis invasivos sobre las piezas- la consulta a catálogos de materiales coloniales publicados por arqueólogos de diversas partes del mundo (Deagan, 1987; Hume, 2001; Schávelzon, 1991) nos permitió resolver, en parte, varias dificultades. En gran medida, porque nos proporcionaron un referente de piezas posibles mucho más amplio que el que nuestra propia experiencia nos permitía reconocer pero, sobre todo, porque más allá de la variabilidad de las materias primas, por principio de homologación pudimos observar semejanzas entre el material de la muestra y material incluido en el catálogo.

Resulta interesante señalar que nuestro propio desconocimiento de la materialidad de ciertos períodos arqueológicos tempranos de nuestro país nos llevó a considerar como potencialmente históricos elementos de culturas tempranas, error que fue subsanado inmediatamente gracias a la colaboración de muchos especialistas que concurren al depósito a consultar material. De hecho, durante todo el desarrollo del trabajo fue permanente la consulta con colegas cuya expertise nos ayudó a reconocer rasgos con los que no estábamos familiarizados, permitiéndonos así afinar paso a paso las definiciones de los criterios propuestos.

La interacción con otros investigadores nos permitió además generar una categoría de piezas "sospechosas "cuyos rasgos no pudieron ser fehacientemente asignados ni por nosotros ni por ellos a uno u otro período. A posteriori este conjunto fue sometido a un análisis detallado a partir de la aplicación de diversos criterios a modo de filtro que buscaron ampliar el referente considerado para su identificación. En algunos casos, ello llevó a descartar definitivamente del catálogo a algunas piezas al reconocerse su carácter prehispánico, mientras que en otros posibilitó identificarlo efectivamente; sin embargo, el grueso del material incluido en esta categoría permanece aún sujeto a revisión.

A fin de organizar la información sobre cada pieza confeccionamos fichas individuales a las que les otorgamos un número interno del catálogo subsanando así, al menos en parte, los problemas asociados a las piezas sin información de origen. En las mismas consignamos aquellos datos que pudiéramos tener de la documentación del museo (número original, colección, procedencia si estuviera registrada y un facsímil de las fichas originales de papel). No obstante, tuvimos que tener especial cuidado al asignar la materia prima, debido a los errores que identificamos en los registros y, por otra parte, al momento de describir las piezas, distinguimos dos instancias: una caracterización tecno morfológica y una asignación funcional propuesta. 
Teoría y Práctica de la Arqueología Histórica Latinoamericana | Año VIII. Vol. 8 | 2019

ISSN en línea: 2591-2801 | ISSN versión impresa: 2250-866X

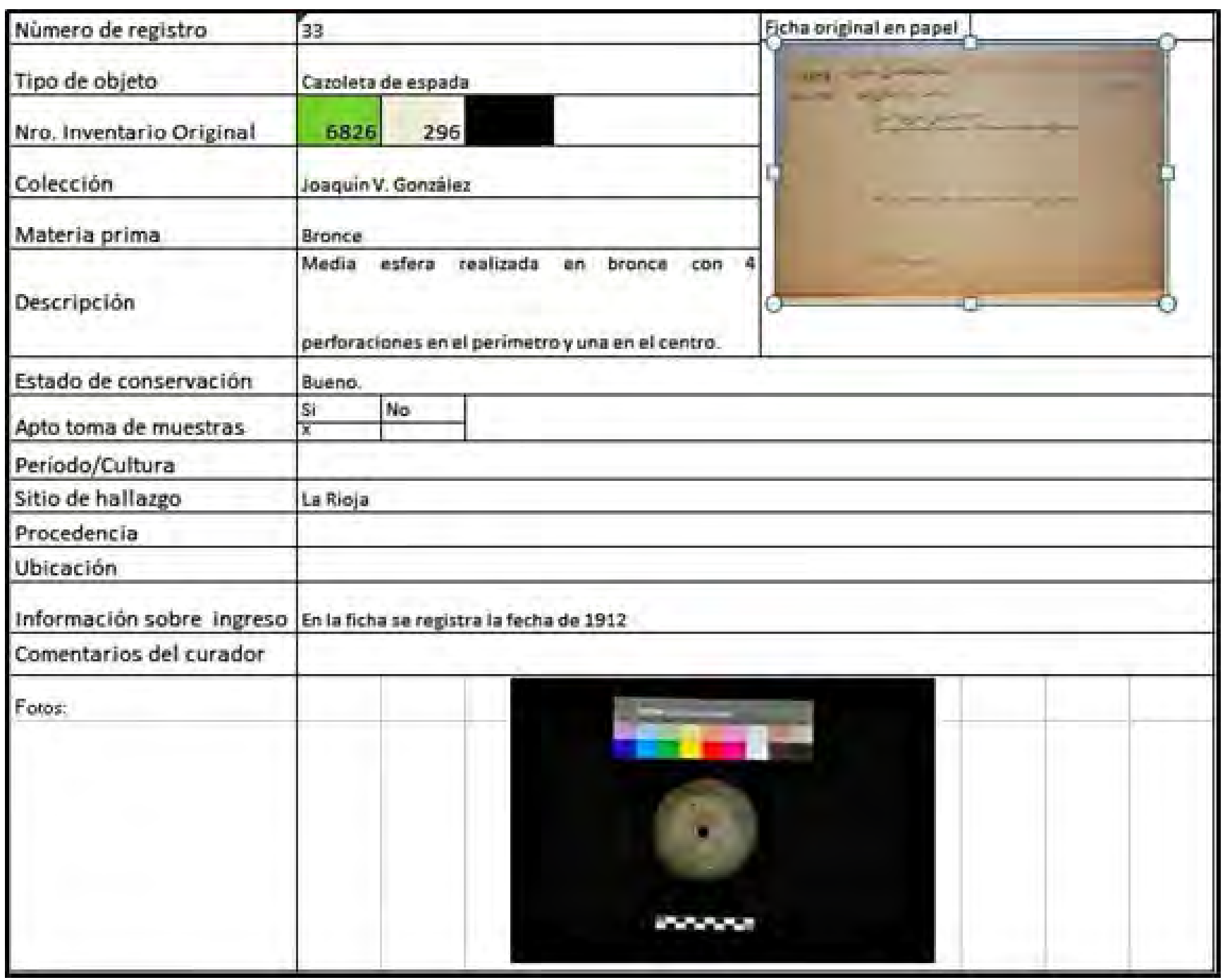



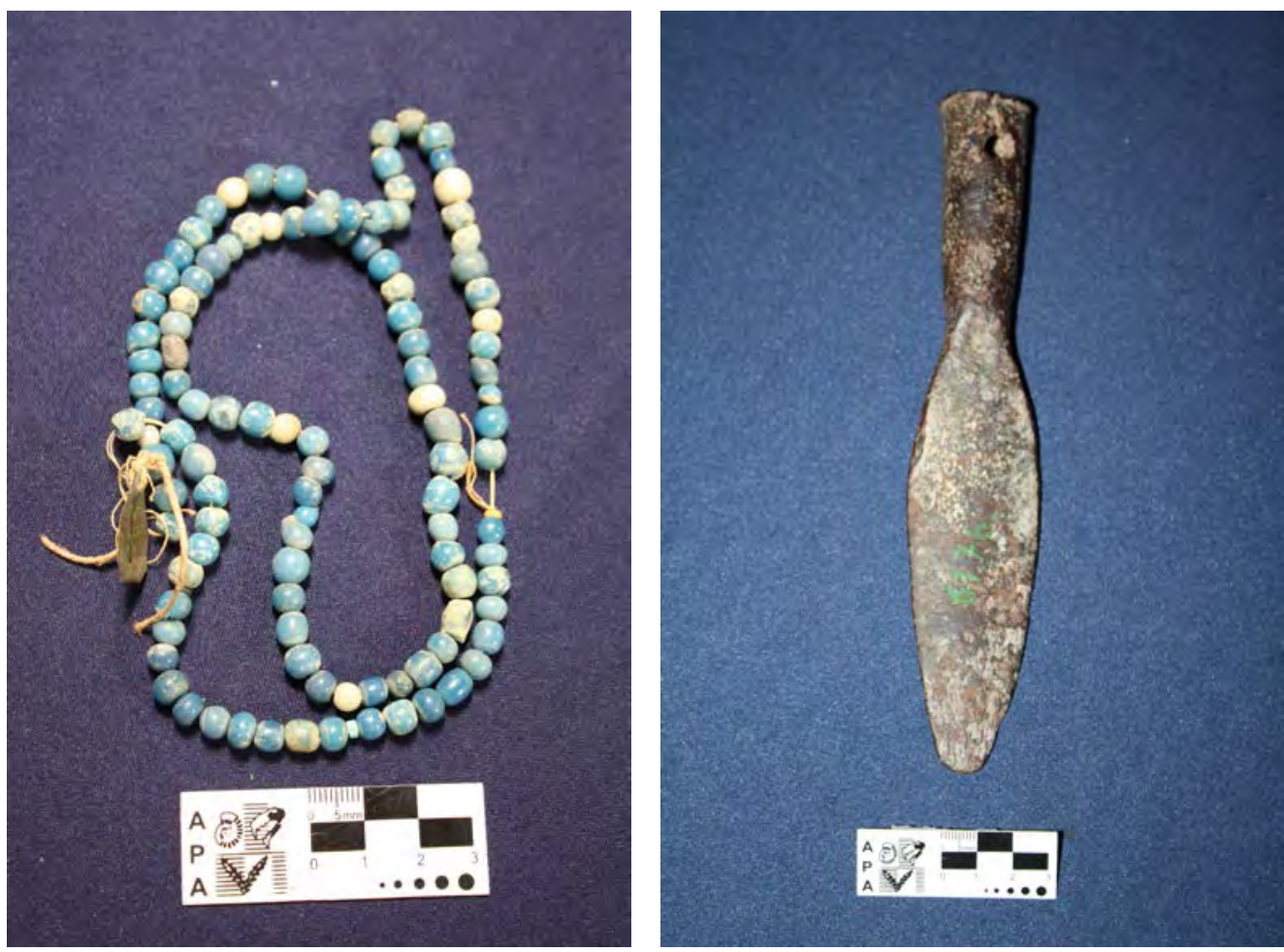

Figuras 2 y 3: Ejemplos de artefactos en vidrio y hierro (collar de cuentas de vidrio -MLP-Ar-(v)5856y punta de lanza de hierro - MLP-Ar-(v)6176- ambas pertenecientes a la Colección Bruch).

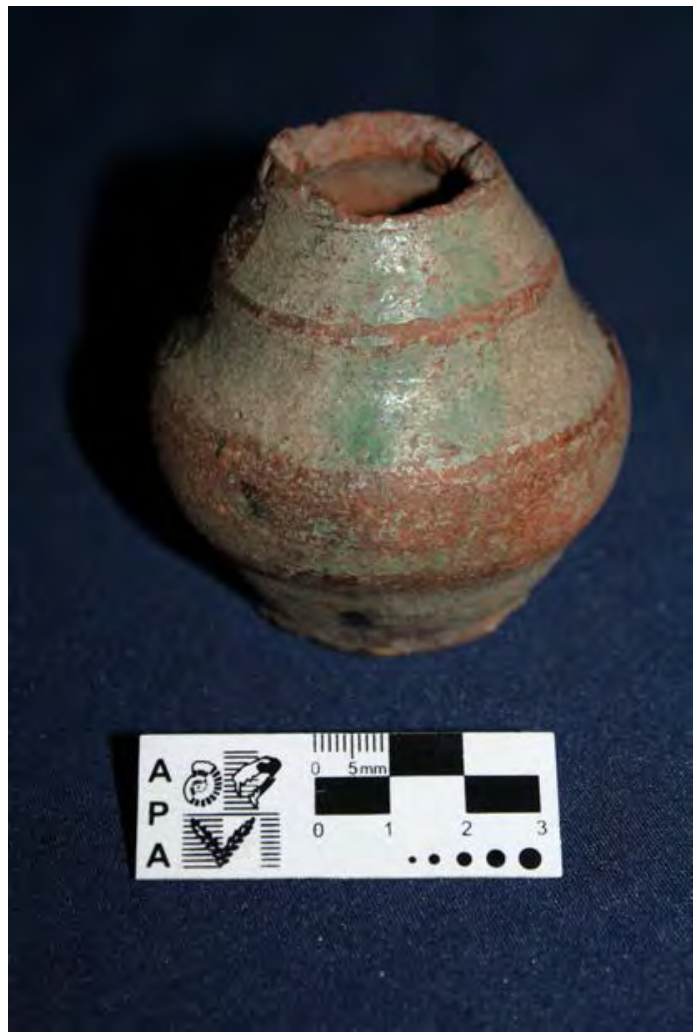

Figura 4: Ejemplo de manufactura con torno y vidriado de superficie (mate -MLP-Ar-(v)6076 - Colección Bruch). 

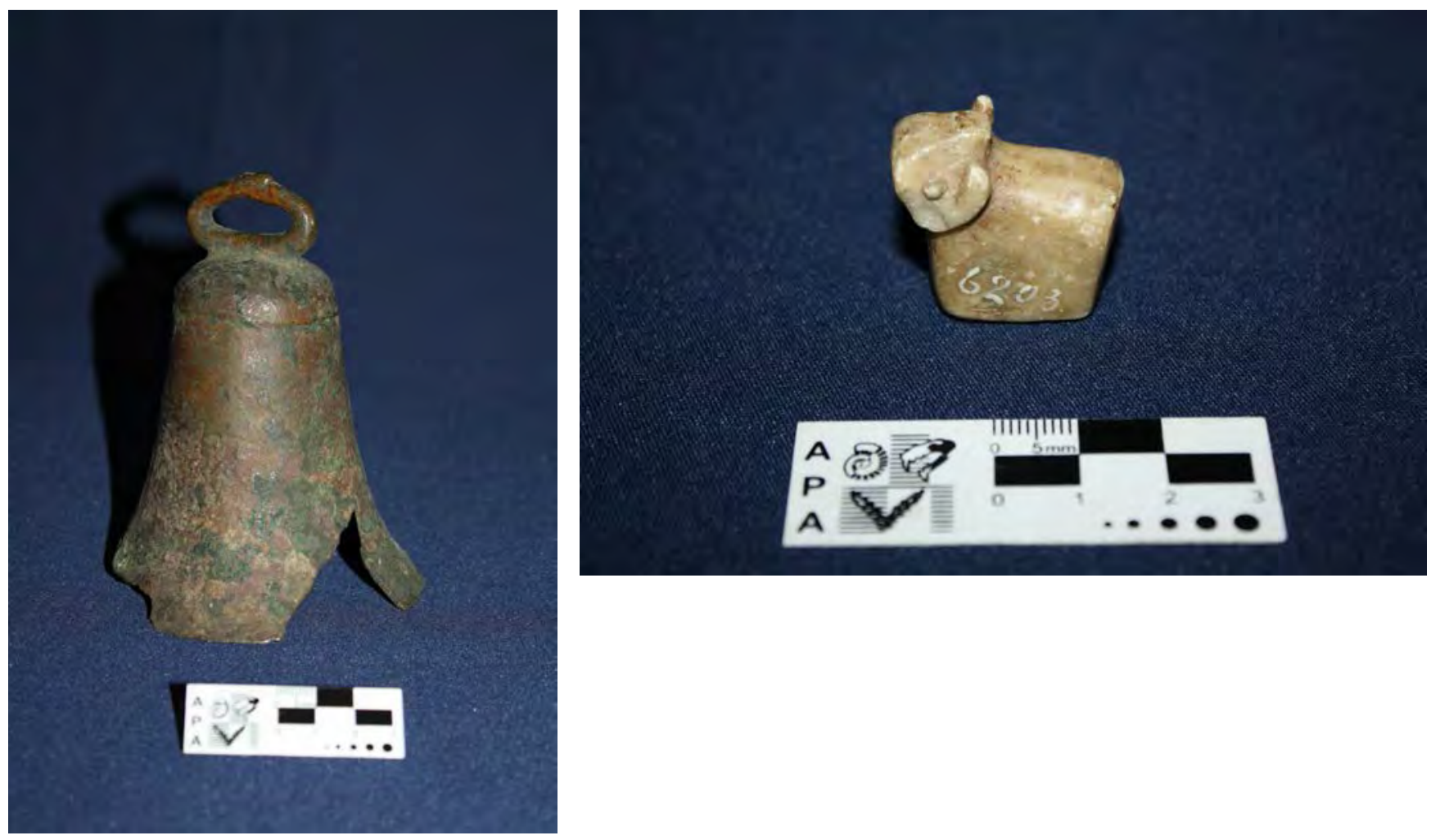

Figuras 5 y 6: Ejemplos de nuevas morfologías (campana de bronce-MLP-Ar-(v)1860- y amuleto tallado con forma de carnero -MLP-Ar-(v)1986- ambos pertenecientes a la Colección Moreno).

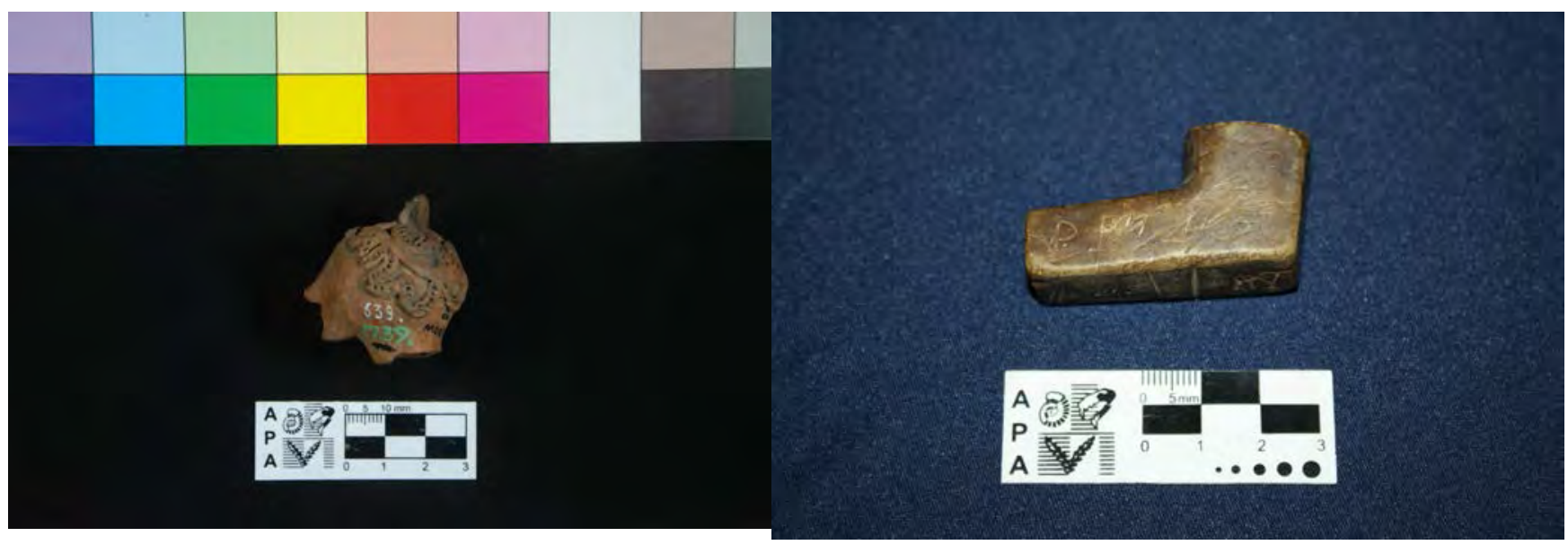

Figuras 7 y 8: Ejemplos de decoración: Peineta y tocado y escritura (Fragmento de figurina -MLP-Ar-(v)1739Colección Moreno- y pipa sin número de identificación) 


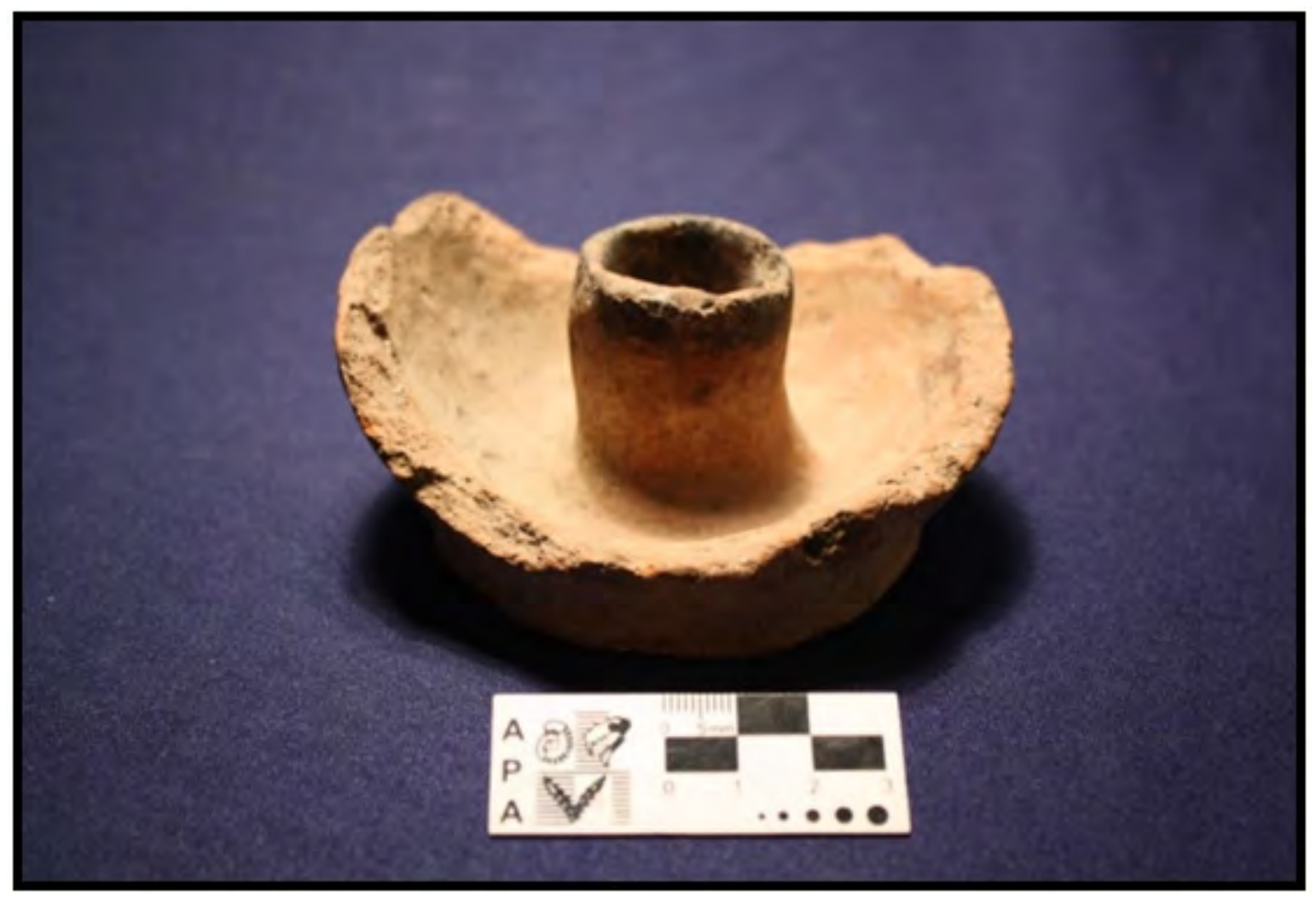

Figura 9: Ejemplo de nueva funcionalidad (Candelabro - pieza sin referencia de colección o procedencia-)

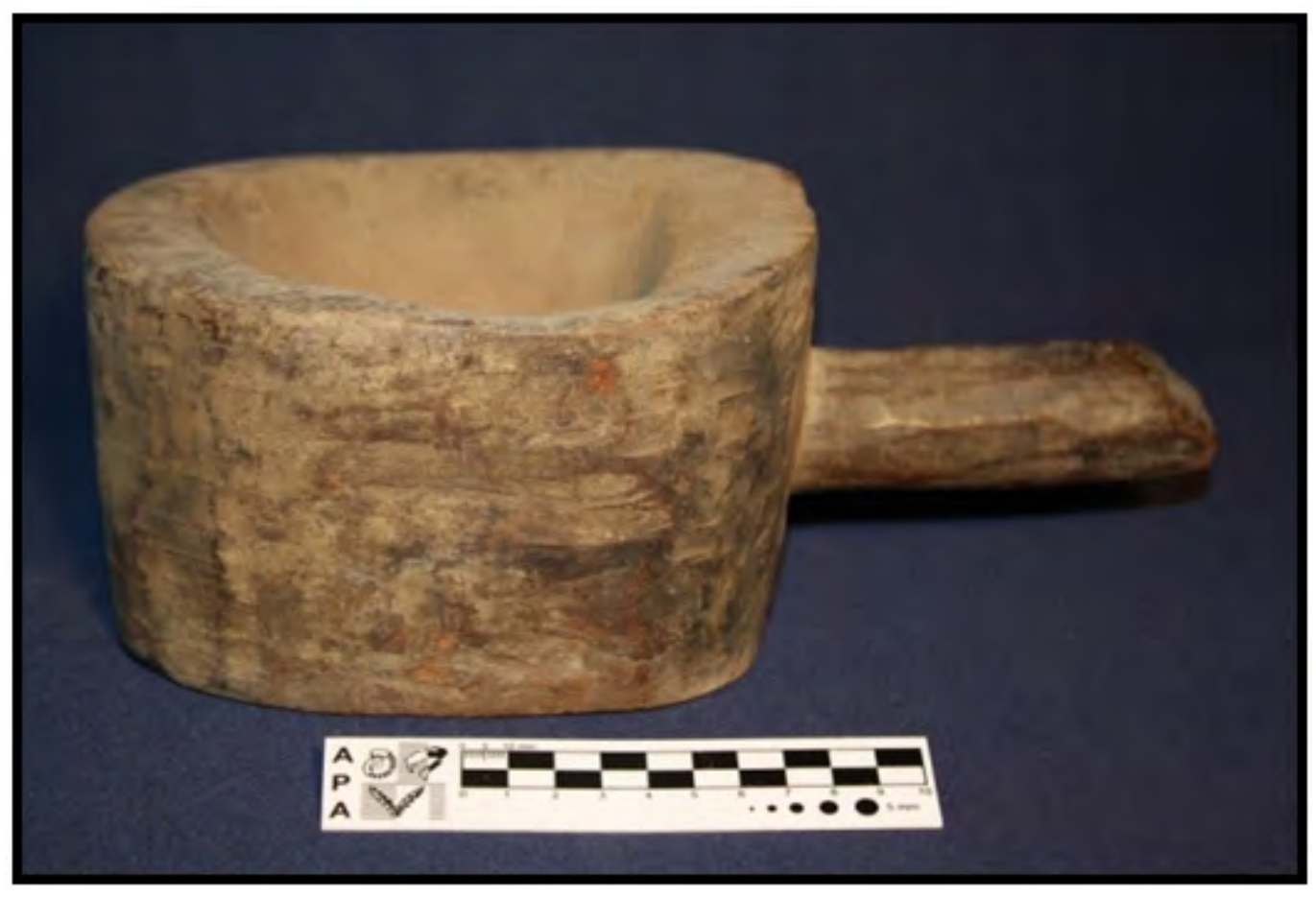

Figura 10: Ejemplo de pieza "sospechosas” (Posible mortero de madera -pieza que procede de la Patagonia, sin referencia de colección-. 
Teoría y Práctica de la Arqueología Histórica Latinoamericana | Año VIII. Vol. 8 | 2019

ISSN en línea: 2591-2801 | ISSN versión impresa: 2250-866X

\section{Discusión}

Al trabajar con piezas de colecciones carentes de información contextual resulta complejo definir qué rasgos nos permiten proponer una asignación cronológica para esa pieza. Durante el proceso de definición de los criterios que pretendemos aplicar al reconocimiento de material histórico dentro de las colecciones recurrimos a algunos utilizados usualmente por otros autores y a otros nuevos propuestos por nosotros a partir de la consulta con otros especialistas y la búsqueda de referentes materiales (publicaciones, catálogos, información biogeográfica y etnohistórica). En la instancia actualmente alcanzada por el desarrollo del trabajo, los únicos atributos que parecen funcionar como criterios diagnósticos con valor absoluto para la identificación del carácter histórico de las piezas son el de materias primas, el de presencia de palabras y/o números claramente reconocibles. En segundo, lugar podemos mencionar que ciertas decoraciones - como los diseños florales y de volutas- algunas técnicas como el vidriado o el torno también presentan una alta confiabilidad al momento de datar las piezas. Sin embargo, entendemos que es la combinación de atributos lo que nos permite afirmar con seguridad que estamos ante un objeto histórico.

Por otra parte, el trabajo de organización de colecciones llevó al surgimiento de preguntas de investigación que se orientaron, en su mayoría, a consideraciones acerca de cómo diferenciar piezas de factura ordinaria prehispánicas o coloniales que pudieran haber sido producidas localmente, y otras semejantes manufacturadas del otro lado del Atlántico, así como también a indagar qué continuidades o rupturas tecnológicas pueden haberse producido como resultado del contacto intercultural ocurrido durante la conquista en piezas de uso doméstico y cómo ello podría verse reflejado en las colecciones analizadas. Dichas situaciones generaron una materialidad difícil de analizar en esta instancia de la investigación sin contextos de referencia.

\section{Consideraciones finales}

Los resultados obtenidos hasta el momento en el marco de un ejercicio de análisis de materiales de colección del Museo permitieron generar una primera herramienta para el estudio de piezas históricas incluidas en otras colecciones o recientemente recuperadas en el terreno, un catálogo de base que registra y organiza sus particularidades. A futuro, se espera ampliar el conjunto estudiado, incorporando nuevas variables de análisis y reajustando la propuesta en función de los nuevos resultados obtenidos por nosotros y por otros investigadores interesados en sumar sus aportes al catálogo. Al igual que otros esfuerzos de clasificación semejantes, el interés último de este trabajo es contribuir a la caracterización del registro material derivado la conformación del nuevo orden sociopolítico en el continente americano a partir de la llegada de los europeos.

\section{Agradecimientos}

Las actividades se realizaron en el marco de un Proyecto Promocional de Investigación y Desarrollo de la Secretaría de Ciencia y Técnica de la UNLP, N018, dirigido por la Dra. Ana Igareta. Queremos agradecer especialmente a todos los investigadores que dedicaron parte de su tiempo a atender nuestras numerosas consultas. 
Teoría y Práctica de la Arqueología Histórica Latinoamericana | Año VIII. Vol. 8 | 2019

ISSN en línea: 2591-2801 | ISSN versión impresa: 2250-866X

\section{Notas}

${ }^{1}$ Cabe recordar que la problemática de la falta de información de las condiciones del hallazgo (situación geográfica exacta, estratigráfica, etc., afecta a un porcentaje significativo de las piezas de colección de la División Arqueología del MLP y los motivos han sido detallados ya en otras publicaciones (Igareta y Collazo, 2011;Igareta y Mariani, 2015; Ghiani Echenique, 2016;Pérez de Micou,1998)

${ }^{2}$ Dos casos ejemplares son el de Carlos Bruch (1911) en el que se incluyen fotografías de diversas locaciones que permiten identificar los lugares recorridos y de esta manera proponer asociaciones con los materiales o el de Delfor Chiappe quién trabajo con la Colección Methffessel.

\section{Referencias bibliográficas}

BRUCH, C.(1911). Exploraciones arqueológicas en las Provincias de Tucumán y Catamarca. Tomo V. Buenos Aires, Argentina. Universidad Nacional de La Plata.

CHIAPPE, D. H. (1962). Estudio arqueológico de la colección Methffessel del Museo de La Plata. (Tesis doctoral). Universidad Nacional de La Plata, La Plata, Argentina.

COLLAZO, J. G. (2012). Colección arqueológica Francisco Pascasio Moreno: pasado, presente y futuro. Cómo conservamos nuestro patrimonio arqueológico. (Tesis de licenciatura en Antropología). Universidad Nacional de Rosario, Rosario, Argentina.

DEAGAN, K. (1987). Artifacts of the Spanish Colonies of Florida and the Caribbean, 1500-1800. Washington D. C., Estados Unidos, Smithsonian Institution Press.

FARRO, M. (2009). La formación del Museo de La Plata. Coleccionistas, comerciantes estudiosos y naturalistas viajeros a fines del siglo XIX. Rosario, Argentina: Prohistoria Ediciones,

GHIANI ECHENIQUE, N. (2016). Buscando en los depósitos. Las colecciones arqueológicas del Museo de La Plata provenientes del litoral rioplatense. La Zaranda de Ideas. Revista de Jóvenes Investigadores en Arqueología, 14 (2), 111-130.

GIAMBELLUCA, R., GIANELLI, J. e IGARETA, A. (2011). Dos colecciones arqueológicas y un destino: el recorrido histórico de la colección Bruch y la colección Lafone Quevedo en el Museo de La Plata. II Simposio de Colecciones de Museos e Investigación, Salta.

HUME, I. (2001).A Guide to Artifacts of Colonial America. Filadelfia, Estados Unidos: University of Pennsylvania Press.

IGARETA, A., BEDNARZ, M. y PENESIS, J. (2018). Piezas históricas en colecciones arqueológicas: criterios aplicados para su identificación. Serie Monográfica y Didáctica: 252 - 253.

IGARETA, A. Y COLLAZO, J. G. (2011). Arqueología de depósito: El potencial informativo de las colecciones del depósito 25 del Museo de La Plata. II Simposio de Colecciones de Museos e Investigación, Salta.

IGARETA, A. Y MARIANI, R. (2015). Acciones de conservación preventiva en depósitos de la División Arqueología del Museo de La Plata. $3^{\circ}$ Encuentro Internacional de Conservación Preventiva e 
Interventiva. Buenos Aires.

IGARETA, A. y VARGAS GARIGLIO. J. (2016). Material vítreo clasificado como lítico en colecciones arqueológicas del Museo de La Plata, Argentina. Antilha Revista Latinoamericana de Historia, Arte y Literatura, 5 (14), 9-25.

PÉREZ DE MICOU, C. (1998). Las colecciones arqueológicas y la investigación. Revista. do Museu de Arqueologia e Etnologia, 8, 223-233.

SCHÁVELZON, D. G. (1991). Arqueología histórica de Buenos Aires. La cultura material porteña de los siglos XVIII y XIX. Buenos Aires, Argentina: Corregidor.

Recibido: 16 de abril de 2019

Aceptado: 01 de julio de 2019 